Cahiers de recherches médiévales

\title{
Figures de la sorcière chez Hugo
}

(Ballades et Notre-Dame de Paris 1482)

\section{Muguras Constantinescu}

\section{(2) OpenEdition Journals}

Édition électronique

URL : https://journals.openedition.org/crm/1773

DOI : $10.4000 / \mathrm{crm} .1773$

ISSN : 1955-2424

Éditeur

Honoré Champion

\section{Édition imprimée}

Date de publication : 15 décembre 2004

Pagination : 111-119

ISSN : $1272-9752$

Référence électronique

Muguras Constantinescu, «Figures de la sorcière chez Hugo », Cahiers de recherches médiévales [En ligne], 11 | 2004, mis en ligne le 10 octobre 2007, consulté le 15 décembre 2022. URL : http:// journals.openedition.org/crm/1773; DOI : https://doi.org/10.4000/crm.1773

Ce document a été généré automatiquement le 15 décembre 2022.

Tous droits réservés 


\title{
Figures de la sorcière chez Hugo
}

\author{
(Ballades et Notre-Dame de Paris 1482)
}

\section{Muguras Constantinescu}

\section{«Il y a tant de sorciers maintenant qu'on les \\ brûle sans savoir leurs noms " \\ Hugo, Notre-Dame de Paris 1482}

$1 \quad$ Parmi les figures mythiques médiévales qui intéressent Victor Hugo dans ses œuvres il y a, sans doute, celle de la sorcière. Comme nous le savons, le Moyen Âge exerce sur le romantique français une grande fascination, fait visible, entre autres, dans ses Ballades et son roman Notre-Dame de Paris 1482. Même si l'auteur place ses deux ouvrages, d'une façon déclarée, sous le signe de l'imagination en général, nous pouvons distinguer entre un type d'imagination poétique, notamment «balladesque » et un autre, épique et romanesque, et par la suite, entre deux manières de figurer la sorcière.

2 Toute figure mythique suppose bien sûr une part d'altération et d'amplification, une «représentation de personnages souvent réels déformés ou amplifiés par l'imagination collective, une longue tradition littéraire », comme le dit le Dictionnaire Robert (1997) et la fiction, en vers ou en prose, ne fait qu'en ajouter, à son tour.

Les Ballades, publiés en 1826, signifient le triomphe de la veine médiévale et l'affirmation d'un imaginaire féerique et magique dans l'œuvre de Victor Hugo. Comme il l'avoue lui-même, il s'agit

des esquisses d'un genre capricieux : tableaux, rêves, scènes, récits, légendes superstitieuses, traditions populaires. L'auteur en les composant a essayé de donner quelque idée de ce que pouvaient être les poèmes des premiers troubadours du Moyen Âge, de ces rhapsodes chrétiens qui n'avaient au monde que leur épée et leur guitare, et s'en aller de château en château, payant l'hospitalité avec des chants. ${ }^{1}$

Dans l'évocation de la sorcière, même fugitive, l'auteur fait tomber l'accent sur le côté légende, superstition, traditions populaires, et cela dans une forte lumière fantastique. Dans sa préface, le poète romantique souligne l'importance de l'imagination dans le cycle proposé par lui - «l'auteur dirait qu'il a mis plus de son âme dans les Odes, plus de son imagination dans les Ballades $»^{2}$. 
5 Le contexte dans lequel est placée la figure de la sorcière est des plus favorables à un épanouissement féerique et magique, parce que Hugo consacre des pièces entières à des figures comme la fée, le sylphe, le lutin, le géant, la péri et dans plusieurs ballades reviennent des images de gnomes, de spectres, de fantômes, de follets, de nains de toutes sortes, de loups rodant, de corbeaux croassant, de démons noirs, et même celle d'un Lucifer volant. Autrement dit, l'arsenal féerique marié à celui de la magie constitue la toile de fond pour tel tableau, tel rêve, tel récit.

6 Le motif du sabbat apparaît plusieurs fois dans le volume par des allusions à des hiboux et à des chauves-souris, "que tout sabbat réclame", ou à la plaine "où le sabbat s'assemble, où les démons hurlants viennent danser ensemble ", préparant de la sorte la ballade consacrée à la fête du diable et de ses acolytes. ${ }^{3}$

7 La pièce qui met en valeur la figure mythique de la sorcière s'intitule La ronde du sabbat et elle se remarque justement par la «verve » avec laquelle est rendue, comme le dit Sainte-Beuve, « l'orgie satanique ». En effet, dans un impressionnant cadre nocturne et funéraire, un monastère-nécropole, le poète imagine la rencontre de Satan avec ses serviteurs dont la sorcière. Là, en illustre compagnie, elle fait partie du cortège de Satan-Lucifer qui comprend larves, dragons, vampires, gnomes, nécromants, démons, lutins, en un mot « des monstres dont l'enfer rêve seul les fantômes »4. C'est, selon le dernier traducteur en date des ballades en roumain, Serban Foarta, un véritable

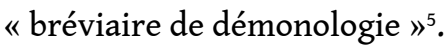

8 La sorcière est présentée dans sa posture emblématique "échappée aux sépulcres déserts / Volant sur le bouleau qui siffle dans les airs " ${ }^{6}$, car sinistre, caricaturale ou même sympathique, l'image de la femme au pouvoir magique, chevauchant un bâton, se perpétue à travers les siècles, en réduisant à l'essentiel et souvent au cliché, tout un imaginaire de la sorcellerie qui nous intéresse ici.

9 L'histoire de la sorcellerie a retenu deux raisons pour lesquelles la sorcière inspirait la peur : d'une part, son pouvoir magique, d'autre part, son pacte avec le diable, la mise à son service ${ }^{7}$. En la présentant dans le cortège de Satan, où elle arrive non pas sur un balai, mais, avec un supplément de mystère et de lien avec la nature, sur un bouleau, Hugo renforce l'image de cette posture spécifique de la sorcière, venue de l'imaginaire médiéval, amplifié et déformé, à sa façon, par le XIXe siècle.

10 Comme nous le laissent croire les spécialistes, le vol sur le balai/bouleau n'est, en fait, qu'un phénomène d'hallucination ; l'absorption de certaines boissons à base de plantes hallucinogènes, l'emploi de certains onguents peuvent produire des hallucinations d'animaux et d'oiseaux et la simulation d'un élan de transvection (Louis, 1999). Selon Brian P. Levack (Villari, 2000), c'est au $\mathrm{XV}^{\mathrm{e}}$ siècle, période qui intéresse particulièrement Hugo, que les accusations de pacte avec le diable sont portées envers des pratiquants ruraux de la magie et non plus envers de savants magiciens. D'ailleurs, ces nouveaux hérétiques sont nommés, à cette époque, sorciers et non plus magiciens. Il s'agit, en général, des gens pauvres, issus des plus basses couches sociales, des sortes de marginaux et surtout des femmes. Le rapport avec le diable change : si les magiciens et surtout les nécromants veulent être les maitres du diable, les sorcières en sont des servantes et des esclaves. C'est justement sur cette attitude de la sorcière que s'arrête le poète dans la pénultième ballade de son cycle.

11 Dans la ronde de sabbat imaginée par Hugo, la figure de la sorcière est seulement esquissée, faisant partie du cortège obligatoire de Satan; elle est présentée comme un 
élément parmi d'autres qui composent l'arsenal démoniaque où le poète place également des éléments rares comme "aspiole» et "psylle» ou obscurs comme "Smarra", justement pour donner du poids et de la couleur à son sabbat. Ceux-ci arrivent en chars tirés par des griffons, sont accompagnés par des nains aux pieds de chèvre, des goules, des femmes infernales, des follets, des spectres blêmes, des boucs méchants, des clercs en magie, des oiseaux fauves.

L'appel de Lucifer s'adresse également à des êtres stigmatisés par les chrétiens comme les Juifs, les Zingaris, les Bohèmes mais l'exhortation d'écrire le mot magique est adressée notamment aux sorcières : « Ecrivez, sorcières : ABRACADABRA ».

Hugo retient seulement quelques aspects du sabbat, en évitant avec une certaine pudeur, le côté érotique qui a développé tout un imaginaire de relations monstrueuses et perverses; il préfère la dimension spectaculaire du sabbat et notamment l'arrivée des serviteurs du diable, l'appel de Satan, la danse qui rythme toute la réunion, la messe tournée en dérision, le signal du départ. Le refrain concernant la ronde diabolique est, selon le même Serban Foarta, comme un « revenant » dans cette ballade des « spectres blêmes » où le poète joue avec le grotesque et les couleurs fortes.

Nous retenons donc de ce premier ouvrage de Victor Hugo à veine médiévale la figure de la sorcière en tant que servante du diable, pratiquant le vol nocturne sur le bouleau, participant à la ronde du sabbat, capable d'écrire le mot au pouvoir magique qui résume l'art démoniaque, tout en soulignant le fait que cette figure déjà mythique est encore transfigurée par l'imaginaire hugolien qui la place dans un cadre terrifiant et parmi des personnages fort grotesques, en amplifiant de la sorte son image et en lui donnant de l'intensité.

Même si le poète ne parle pas ouvertement de sorcière, il y a dans ses Ballades encore une référence qui renvoie à la figure de la sorcière, telle qu'elle sera évoquée plus tard dans le roman; il s'agit de la pièce La fiancée du timbalier, où fait son apparition une " égyptienne sacrilège ", " vieille au regard de vipère » qui, par son don de prédiction, annonce à la jeune fille la mort de son fiancé; sa figure est associée à un cadre maléfique, "derrière un pilier ", près d'un "sépulcre, son noir repaire » et elle est envisagée de manière nettement négative. Par son don de prédire le sort, la vieille égyptienne rappelle le sens étymologique du terme "sorcier, ière " (XII ; de sorcerius VIII ${ }^{\text {e }}$ du lat. pop. sortiarius, " diseur de sorts ", lui-même du lat. sors), ayant donc le sens de «personne qui pratique une magie de caractère primitif, secret et illicite (sorcellerie)».

Dans le roman à sujet médiéval Notre-Dame de Paris 1482, la figure de la sorcière, ou plutôt de la jeune fille accusée de sorcellerie, occupe une place centrale. Même si ce roman médiéval de Victor Hugo est souvent considéré comme un roman historique, l'auteur, dans une lettre adressée à son éditeur, renie cette étiquette: «Le livre n'a aucune prétention historique, si ce n'est de peindre peut-être avec quelque science et conscience... l'état des mœurs, des croyances, des lois, des arts, de la civilisation enfin, au quinzième siècle». Son affirmation nous intéresse justement par la partie concernant «l'état des mœurs, des croyances, des lois» (Hugo, op. cit., p. XXII), car la sorcière et la sorcellerie y sont vues sous ces multiples aspects. Il y a une part de reconstitution historique, de chronique d'époque qui place la figure de la sorcière dans une période de l'histoire bien déterminée (n'oublions pas que le titre complet du roman contient cette précision chronologique, 1482), la fin du Moyen Âge et le début d'une 
époque moderne, le moment où la rivalité entre l'édifice et le livre va se résoudre en faveur du dernier.

Quelques repères de l'histoire de la sorcellerie nous aident à mieux comprendre l'histoire d'Esmeralda, la prétendue sorcière, autour de qui tournent les autres personnages du roman.

Selon Jacques Le Goff et Jean-Claude Schmitt (1999), au Moyen Âge la sorcellerie garde encore des restes de l'idolâtrie païenne, exprimée par des charmes, sorts, divination, magie, nécromancie, réprimés comme antichrétiens. Elle va s'amplifier progressivement, étant associée et assimilée à l'hérésie. Ce processus montre, de façon exemplaire, comment l'imaginaire, le fantasme, la croyance peuvent être des forces historiques véritables. L'importance de la sorcellerie vient du fait qu'elle repose sur des croyances partagées jusqu'au sommet des institutions dominantes de l'Église et de l'État ce qui va conduire à sa dure répression.

La sorcellerie est, en fait, une modalité d'interpréter le monde, par l'attribution des malheurs au diable et à ses complices ce qui distingue, d'une part, les institutions, d'autre part, le diable et les sorcières. En ce qui concerne la « chasse aux sorcières » qui a été déclenchée en Europe entre le $\mathrm{XV}^{\mathrm{e}}$ siècle et la première moitié du XVIII ${ }^{\mathrm{e}}$ siècle, le Moyen Âge est seulement une période de genèse, genèse qui s'explique notamment par l'élargissement des compétences de l'Inquisition à juger non seulement les hérésies mais aussi la sorcellerie. On peut dire qu'en l'absence de l'Inquisition et de la torture la chasse aux sorcières n'aurait pas été déclenchée (Le Goff, 1999).

Il faut retenir aussi de ces quelques repères d'une histoire de la sorcellerie l'intensité particulière de la chasse aux sorcières en Europe après 1430 et jusque 1498, justement dans la période envisagée par Victor Hugo dans son roman.

Pour revenir à Esméralda, la sorcière du roman est une égyptienne, une bohémienne, une marginale qui mène sa vie parmi des truands, en étant leur " Notre-Dame ", leur "sœur "; enfant trouvé ou volé, orpheline à la recherche de ses parents, elle vit en compagnie d'une chèvre dressée qui lui assure un numéro de succès sur la place publique; être primitif, Esméralda comprend le langage de la bête et la considère comme sa "sœur "; c'est, comme dit Eugène Sue, "une jeune fille naïve, presque sauvage au milieu de la civilisation $»^{8}$. Elle croit au pouvoir de son amulette pour l'aider à retrouver ses parents et, sans doute, aux pouvoirs des pierres précieuses, notamment celles de l'émeraude, même si elle n'en a qu'une simple imitation en verre. Ses pratiques et croyances tiennent donc de la plus bénigne magie blanche, ou même des simples superstitions et traditions populaires.

Telle que la présente le narrateur, c'est une jeune fille de seize ans, innocente, naïve dont la seule faute est d'exercer fascination et séduction sur son public et notamment sur trois ou quatre hommes, personnages importants dans cette histoire d'imagination car, nous n'allons pas oublier l'importance de l'imagination dans ce roman « historique »: «S'il a un mérite, c'est d'être une œuvre d'imagination, de caprice et de fantaisie » (Hugo, op. cit., p. XXII).

Le début du roman montre justement l'attraction qu'exerce La Esmeralda par sa danse, accompagnée de sa propre musique au tambourin sur la place publique; il s'agit d'un spectacle populaire, dans la tradition des spectacles forains, courants à l'époque où Hugo place son histoire. Sa beauté séduisante, son art de danser et de communiquer avec sa chèvre assurent à la jeune fille une renommée qui précède son entrée en scène 
(" Mais je veux que le diable m'écorche si je comprends ce qu'ils veulent dire avec leur Esmeralda ! Qu'est-ce que c'est que ce mot-là d'abord ? C'est de l'égyptiaque ! $\left.»^{9}\right)$.

Sa première apparition est à la hauteur de sa renommée car, en la voyant, Pierre Gringoire croit avoir affaire à quelque être fantastique : " une surnaturelle créature », « une salamandre, une déesse, une nymphe, une bacchante du Mont-Ménaléen $»^{10}$.

Comme Hugo veut, sans doute, démontrer par son histoire comment une fille naïve, innocente, vertueuse et généreuse peut être accusée de sorcellerie à cause de son statut de marginale (égyptienne, bohémienne, orpheline, "sœur » des truands), il accentue les contrastes jusqu'au paradoxe, car aux yeux du poète Gringoire la première impression est celle d'un "ange " et d'une "fée ", aux yeux du narrateur l'impression est celle d'une "céleste créature », lumineuse : "d'une beauté si rare [...] il sembla qu'elle y répandait une sorte de lumière qui lui était propre $"^{11}$.

Sa beauté, concentrée en trois éléments typiques, selon une longue tradition, pour le portrait féminin - cheveux noirs, peau brune, yeux de flamme - est complétée par sa vivacité, son agilité, car elle est vue comme "folle», "vive», "mince», "frêle», " agile ", "légère ", " joyeuse ", par les divers spectateurs dont le narrateur. Son apparence et le spectacle qu'Esmeralda donne avec sa chèvre qui sait imiter des personnages illustres dépassent l'ordinaire et éblouissent, suggérant déjà l'idée de magie : « le tableau n'était pas sans prestige et sans magie ${ }^{12}$. Quand celui qui regarde est animé par la haine et la passion sombre, comme c'est le cas de l'archidiacre Frollo, il ne s'agit plus de magie mais de sa forme altérée et maléfique, la sorcellerie : «il y a de la sorcellerie là-dedans ", « sacrilège, profanation " $^{13}$.

En fait, toute l'histoire conçue par Hugo montre, avec les moyens de l'écrivain, comment mentalité, préjugés, ressentiment, refoulement, intolérance, imaginaire individuel ou collectif transforment une danseuse des rues en sorcière, comment les masses populaires peuvent être facilement manipulées par les institutions du pouvoir au point de passer de l'admiration devant la beauté et le talent d'une jeune fille ( $L a$ Esmeralda! La Esmeralda dans la place! Ce mot produisit un effet magique. Tout ce qui restait dans la salle se précipitait aux fenêtres, grimpant aux murailles pour voir, et répétant: la Esmeralda! la Esmeralda! $\left.»^{14}\right)$ à la demande frénétique de son exécution ("Ces flambeaux semblaient chercher quelque chose. Et bientôt ces clameurs éloignées arrivèrent distinctement jusqu'aux fugitifs :- L'égyptienne! la sorcière!, à mort l'égyptienne! $\left.»^{15}\right)$.

Il faut remarquer que toute l'histoire est vue avec le regard, la "science " et la » conscience ", comme dit l'auteur lui-même, d'un homme du XIX ${ }^{\mathrm{e}}$ siècle, des temps modernes, qui analyse avec une distance de «trois cent quarante-huit ans six mois et dix-neuf jours » les événements qu'il relate. La distanciation de l'auteur est soulignée à plusieurs reprises et elle prend parfois la forme de la reconstitution historique à base de documents, ouvrages, érudition.

D'ailleurs en matière de sorcières et sorcellerie, on a toujours, comme le remarquent les spécialistes, le regard des accusateurs et moins ceux des accusés. Victor Hugo essaie par sa fiction de montrer les deux, en les plaçant dans une forte opposition.

Dans la première partie, nous assistons aux succès et au rayonnement de la jeune danseuse, dans la deuxième partie nous voyons sa poursuite, sa condamnation, sa stigmatisation. Déjà, dès sa première apparition, malgré la sympathie et l'admiration du grand public, la jeune danseuse a contre elle le sombre vierge Frollo, partagé entre 
haine et passion interdite, et la folle recluse qui déteste les égyptiens parce qu'elle suppose qu'ils lui ont volé l'enfant et même qu'ils le lui ont mangé. On voit déjà dans la hantise de la recluse comment l'imagination, maladive en son cas, et un ressentiment personnel, peuvent amplifier et transfigurer la perception des faits jusqu'à y ajouter du cannibalisme.

31 Si dans le cas de l'archidiacre et de la Sachette, on a affaire à un côté pathologique, individuel, aux cas des jeunes filles de "bonne maison », possibles " accompagneresses d'honneur de la Dauphine Marguerite ", il s'agit de préjugés sociaux, raciaux de caste, auxquels s'ajoutent aussi jalousie et rivalité féminines. Tout ce qui est différence par rapport à leurs normes et goûts est ressenti comme ridicule et suspect. Le nom " étrange », Esmeralda, " prodigieusement sarrasin » selon Phoebus, provoque un fou rire chez les jeunes aristocrates qui, rien qu'à cause de son nom, voient dans la jeune danseuse, progressivement, une «charmeresse», une "magicienne», une "bohémienne de l'enfer " ${ }^{16}$.

Il faut dire d'ailleurs qu'un riche paradigme est déployé tout au long du roman pour nommer la figure de la sorcière ; on peut retenir ainsi à côté des mots déjà mentionnés, des termes comme "bohème", "bohémienne", "égyptienne", «égyptienne du démon », « sauterelle d'Egypte ", « cigale d'enfer », " gitane », « zingara », « effroyable stryge », ce dernier étant un mort très fort parce qu'il veut dire « vampire tenant de la femme et de la chienne ».

33 Tandis que le poète-saltimbanque, plutôt son frère et son ami que son mari, voit dans Esmeralda une "créature inoffensive et charmante, jolie [...]; une fille naïve et passionnée, ignorante de tout; ne sachant pas encore la différence d'une femme à un homme, même en rêve; faite comme cela; folle surtout de danse, de bruit, de grand air ; une espèce de femme abeille, ayant des ailes invisibles aux pieds, et vivant dans un tourbillon. Elle devait cette nature à la vie errante qu'elle avait toujours menée ${ }^{17}$, l'archidiacre, trop tenté par elle, la voit, comme toute l'Église qu'il représente, en tant qu' égyptienne du démon » dont le contact « vous ferait vassal de Satanas »"

Si dans le deuxième chapitre du septième livre, la jeune Esmeralda qui ne pratique que la danse et le dressage de sa chèvre, peut être considérée «à l'abri de ces procès de magie si fréquemment intentés aux bohémiennes » qui disent la «bonne aventure ${ }^{19}$, si, quelques chapitres plus loin, dans la scène avec Phoebus, elle le met mal à l'aise par son "parfum de chasteté » et son " charme de vertu », après l'assassinat du capitaine par le prêtre en la présence de la jeune fille, avant même que le procès commence, l'étiquette de «sorcière » lui est vite collée : « elle entendait dire autour d'elle : C'est une sorcière qui a poignardé un capitaine $»^{20}$.

Le procès est l'occasion de voir comment s'épanouit l'imaginaire de la haine et du ressentiment, dans la confusion, sous l'influence de la version altérée des accusateurs, représentant la loi et l'église et, bien sûr, l'idéologie dominante. Ainsi, l'entremetteuse Falourdel, principal témoin, se souvient d'une fille belle comme une poupée, mais à la place d'une chèvre aux cornes dorées, elle se rappelle un "grand bouc noir ou blanc ${ }^{21}$; dans cette deuxième accusée, la chèvre "ensorcelée ", on identifie vite le diable et dans la gracieuse, autrefois, danseuse du carrefour une " effroyable stryge $»^{22}$.

Comme le procès d'un animal peut paraître au lecteur du XIX ${ }^{e}$ siècle un peu extravagant, l'auteur intervient avec un commentaire digressif, des documents à 
l'appui, pour donner une idée de l'ampleur et de l'ordinaire du phénomène au Moyen Âge :

Rien de plus simple alors qu'un procès de sorcellerie intenté à un animal. On trouve, entre autres, dans les comptes de la prévôté pour 1466, un curieux détail des frais du procès de Gilles-Soulart et de sa truie, exécutés pour leurs démérites, à Corbeil [...]. Quelquefois même on allait plus loin que les bêtes. Les capitulaires de Charlemagne et de Louis le Débonnaire infligent de graves peines aux fantômes enflammés qui se permettaient de paraître dans l'air. ${ }^{23}$

Le récit du procès est également une illustration du fait que le sabbat et la sorcellerie étaient souvent œuvre d'érudition, tout comme les fantasmes des juges et des inquisiteurs qui, en interrogeant les prétendues sorcières, les « instruisent » en quelque sorte (Louis, 1994). Ainsi, par exemple, on demande, sous torture, à Esmeralda, de reconnaître que « vous avez vu le bélier que Belzébuth fait paraitre dans les nuées pour rassembler le sabbat, et qui n'est vu que des sorciers $»^{24}$. Une autre accusation, toute aussi fantasmatique, fait le lien avec la ballade évoquée plus haut pour son pittoresque sombre et son érudition démonologique: "Jeune fille bohème, vous avouez votre participation aux agapes, sabbats et maléfices de l'enfer, avec les larves, les masques et les stryges? $»^{25}$.

L'accusation finale de "crime de sorcellerie, de magie, de luxure et de meurtre $»^{26}$ semble naturelle après cet interrogatoire truffé de références savantes adressé à une jeune fille peu instruite mais qui est considérée d'emblée « de race bohème, adonnée aux maléfices $»^{27}$.

Retenons également comme accusation contre Esmeralda la prétendue transformation, en complicité avec les " puissances des ténèbres ", de l'écu en feuille de bouleau (l'arbre sur lequel chevauche la sorcière de la Ronde du sabbat), qui aux yeux des juges est une "nouvelle preuve de magie », à cause aussi du type d'arbre d'où la feuille provient ${ }^{28}$.

Comme nous le savons, dès le scénario initial de 1830, était prévus le procès de la sorcière et de la chèvre, la condamnation et l'amende honorable au portail, tout comme la décision du roi Louis XI « qu'on charge la populace et qu'on pende l'Egyptienne ${ }^{29}$; cela nous montre l'intérêt vraiment capital que le romancier accorde à la sorcellerie et à sa répression comme phénomène spécifique et dévalorisant d'un Moyen Âge séduisant, par ailleurs, par son architecture et son art.

41 Mais outre la figure de la prétendue sorcière il y a dans le roman de Victor Hugo au moins deux figures masculines de la sorcellerie dans l'archidiacre Frollo et le bossu Quasimodo, l'un se trouvant vis-à-vis de l'autre dans un rapport de paternité adoptive, l'autre de chien obéissant à son maître. Il s'agit à nouveau de l'imaginaire collectif qui cherche à caser un être différent dans une catégorie connue et dévalorisante pour l'apprivoiser en quelque sorte.

42 Frollo s'attire l'étiquette de sorcier par son aspect et son comportement étranges et, à la fois, par ses intérêts pour l'alchimie; au moment où il adopte l'enfant monstrueux qu'il va nommer Quasimodo, les femmes qui assistent à cette scène le considèrent comme un sorcier, parce que seul un être bizarre, un sorcier peut prendre une telle décision, incompréhensible pour les gens ordinaires. La cellule où Frollo, en posture faustienne, fait ses expériences alchimiques, est vue par les autres comme une « logette aux sorcelleries", une "cuisine d'enfer $»^{30}$. Le même étrange archidiacre va être assimilé au démon et ensuite au "moine-bourru", sorte de fantôme maléfique qui hante, le soir, les rues de Paris. Mais une fois tombé amoureux d'Esméralda, 
« resplendissante figure », d'une "beauté surhumaine »" , il se sent «ensorcelé »et y reconnaît une autre magie que celle qu'il connaît et qui lui échappe.

À cause de sa monstruosité physique, de son étrangeté, de sa différence, en fin de compte, le bossu Quasimodo est assimilé lui aussi à un sorcier, "faiseur de charmes ", " chevaucheur de manche à balai », "chat huant", «jeteur de sorts" du haut de ses tours, qui a le mauvais œil et le pouvoir de métamorphoser en monstres les enfants dans le ventre de leurs mères.

Nous pouvons dire, en guise de conclusion, que la figure mythique de la sorcière et la sorcellerie médiévales préoccupent Victor Hugo dans ses Ballades et dans son roman Notre-Dame de Paris 1482. Il nous en donne quelques illustrations très fortes et très convaincantes qui montrent la part très importante de l'imaginaire soit populaire, soit érudit dans la stigmatisation d'une figure bénigne pour l'essentiel.

Il reconstitue, en s'appuyant sur des documents et des chroniques, un cadre médiéval favorable à la transformation de la sorcière, prétendue ou réelle, bénéfique ou maléfique, en figure mythique, vue avec le regard de l'homme moderne qui marie avec bonheur imagination et fantaisie, science et conscience.

\section{BIBLIOGRAPHIE}

Dominique Camus, Pouvoirs sorciers, Paris, Imago, 1998.

Jean-Paul Clébert, Bestiar fabulos, Bucuresti, Artemis-Cavallioti, 1995.

Serban Foarta, Glose in Victor, Hugo, Ballades-Balade, Tîrgoviste, Editura-M, 2002.

Victor Hugo, Odes et ballades, Les Orientales, Paris, Garnier-Flammarion, 1968.

- Ballades-Balade, Tîrgoviste, Editura-M, 2002, trad., glose Serban Foarta.

- Notre-Dame de Paris, Paris, Le Livre de Poche, 1998.

- Notre-Dame de Paris : 1482, Paris, Pockett, 1989.

Jacques Le Goff, Jean-Claude Schmitt, Dictionnaire raisonné de l'Occident médiéval, Paris, Fayard, 1999.

Brian P. Levanck, Vrajitoarea in Rosario, Villari Omul baroc, Iasi, Polirom, 2000.

René Louis, Dictionnaire du mystère, Paris, Editions du Félin, 1994.

Rosario Villari, Omul baroc, Iasi, Polirom, 2000.

\section{NOTES}

1.Victor, Hugo, Odes et ballades, Les Orientales, Paris, Garnier-Flammarion, 1968, p. 303.

2.Ibid.

3.Victor, Hugo, Ballades-Balade, Tîrgoviste, Editura-M, 2002, trad. et glose Serban Foartan p. 64 et p. 80.

4.Ibid., p. 136. 
5.Ibid, p. 174 .

6.Ibid., p. 136.

7.Rosario, Villari, Omul baroc, Iasi, Polirom, 2000, p. 257.

8.Victor Hugo, Notre-Dame de Paris 1482, Paris, Pockett, 1989, p. XXIII.

9.Ibid., p. 83.

10.Ibid., p. 94.

11.Ibid., p. 299.

12.Ibid., p. 95.

13.Ibid., p. 96.

14.Ibid., p. 82.

15.Ibid., p. 554.

16.Ibid., p. 304-305.

17.Ibid., p. 312.

18.Ibid., p. 314.

19.Ibid., p. 313.

20.Ibid., p. 356 et 362.

21.Ibid., p. 368.

22.Ibid., p. 373.

23.Ibid., p. 372.

24.Ibid., p. 379.

25.Ibid.

26.Ibid., p. 382.

27.Ibid., p. 373.

28.Ibid., p. 370 .

29.Ibid., p. 616 .

30.Ibid., p. 322.

31.Ibid., p. 392.

\section{AUTEUR}

\section{MUGURAS CONSTANTINESCU}

Universitatea Stefan cel Mare, Roumanie 